\title{
Efficient Variable Step Size Approximations for Strong Solutions of Stochastic Differential Equations with Additive Noise and Time Singularity
}

\author{
Harry Randolph Hughes and Pathiranage Lochana Siriwardena \\ Department of Mathematics, Southern Illinois University Carbondale, 1245 Lincoln Drive, Carbondale, IL 62901, USA \\ Correspondence should be addressed to Harry Randolph Hughes; hrhughes@siu.edu
}

Received 20 December 2013; Revised 27 May 2014; Accepted 10 June 2014; Published 2 July 2014

Academic Editor: M. Jesus Lopez-Herrero

Copyright (C) 2014 H. R. Hughes and P. L. Siriwardena. This is an open access article distributed under the Creative Commons Attribution License, which permits unrestricted use, distribution, and reproduction in any medium, provided the original work is properly cited.

\begin{abstract}
We consider stochastic differential equations with additive noise and conditions on the coefficients in those equations that allow a time singularity in the drift coefficient. Given a maximum step size, $h^{*}$, we specify variable (adaptive) step sizes relative to $h^{*}$ which decrease as the time node points approach the singularity. We use an Euler-type numerical scheme to produce an approximate solution and estimate the error in the approximation. When the solution is restricted to a fixed closed time interval excluding the singularity, we obtain a global pointwise error of order $O\left(h^{*}\right)$. An order of error $O\left(h^{*} p\right)$ for any $p<1$ is obtained when the approximation is run up to a time within $h^{* q}$ of the singularity for an appropriate choice of exponent $q$. We apply this scheme to Brownian bridge, which is defined as the nonanticipating solution of a stochastic differential equation of the type under consideration. In this special case, we show that the global pointwise error is of order $O\left(h^{*}\right)$, independent of how close to the singularity the approximation is considered.
\end{abstract}

\section{Introduction}

Numerical approximation methods for stochastic differential equations (SDEs) are well developed for SDEs with coefficients satisfying Lipschitz conditions. One of basic methods is the Euler-Maruyama algorithm which is presented in [1] and also well explained by Kloeden and Platen [2]. It is developed using Taylor series expansions and the Itô formula and is analogous to the Euler algorithm for the deterministic case. The Euler method with constant step sizes gives the global error $O\left(h^{1 / 2}\right)$ when drift and diffusion terms satisfy global Lipschitz and linear growth conditions. In addition to methods with constant step sizes, methods with variable (adaptive) step sizes have been developed, for example, those in [3-7]. In general, these methods seek to improve the efficiency of the algorithms by adapting the step sizes to the state of the numerical solution at successive stages of the algorithm.

We consider stochastic differential equations with additive noise and an endpoint singularity with respect to the time variable in the drift term. With this time singularity, the global error estimate for the Euler-Maruyama scheme can only be applied up to a fixed time before the singularity. Using fixed step sizes to generate a numerical approximation for a time closer to the singularity comes at a great cost in efficiency due to the much smaller step size required to produce the same order of global error. We seek to increase the efficiency of the algorithm by using variable step sizes adapted to the shape of the singularity. In making this adaptation, we must also ensure that the step sizes do not become so small that the sum of the steps cannot reach times arbitrarily close to the singularity.

We consider a stochastic differential equation of the form

$$
\begin{gathered}
d X_{t}=\frac{g\left(t, X_{t}\right)}{1-t} d t+\sigma d W_{t}, \quad 0 \leq t<1, \\
X(0)=x_{0},
\end{gathered}
$$

where $\sigma$ is a constant, $W_{t}$ is standard Brownian motion, and $g(t, x)$ satisfies linear growth and Lipschitz conditions with 
bounds for derivatives up to the second order. For example, suppose that, uniformly in $t$ on $[0,1)$,

$$
\begin{gathered}
|g(t, x)| \leq k_{0}(1+|x|), \\
\left|g_{1}(t, x)\right| \leq k_{1}(1+|x|), \\
\left|g_{2}(t, x)\right| \leq C_{1}, \\
\left|g_{22}(t, x)\right| \leq C_{2}, \\
\mathbb{E}\left[X_{t}^{2}\right] \leq K,
\end{gathered}
$$

where $g_{i}$ denotes the partial derivative of $g$ with respect to the $i$ th variable.

Note that the singularity in the drift term stops us from using the standard results of numerical analysis. To overcome this problem, we use variable step sizes and stop the approximation at $t=1-\delta$ where $0<\delta<1$. We estimate bounds for the pointwise local error and we use a lemma [8] to estimate the $L^{2}$ global error. We fix a maximum step size $h^{*}$ and produce variable steps and node points depending on $h^{*}$ in order to produce an estimate of the global error of order $O\left(h^{*}\right)$ for fixed $\delta$. The same algorithm and analysis using fixed step sizes fail to produce an estimate of global error. We further show that there is a choice of $\delta$ depending on $h^{*}$ so that the global error is of order $O\left(h^{*}\right)$ for positive $p$ and $\delta$ converges to 0 as $h^{*}$ converges to 0 . In this analysis we also obtain an estimate of the number of node points required for a given $h^{*}$ and $\delta$. The trade-off for producing our global error estimate is that this number grows faster than $1 / h^{*}$, the corresponding number of node points for fixed step size approximations.

In Section 4, we apply this method to the process well known as the Brownian bridge (BB). Here we use the version of Brownian bridge that is the strong, adapted solution of a stochastic differential equation of the form (1a)-(1b) [9, p. 75]. $\mathrm{BB}$ is a stochastic process that starts at a specific point and converges to a specific point at a given time. $\mathrm{BB}$ is widely used in Monte Carlo simulations and many other applications [10, 11]. Most of the applications use simpler anticipating versions of BB like $X_{t}=W_{t}-t W_{1}$ to avoid the approximation problem that occurs from the singularity in the drift term. We apply our methods to numerically approximate Brownian bridge and obtain even better estimates of global error than those for our general problem. For more properties of Brownian bridge, one can refer to [12, p. 86-89].

Brownian bridge from $a$ to $b$ is given by the following SDE [9, p. 75]. For the sake of simplicity, we use $a=0$ and $b=1$ later in our analysis. For fixed $a, b \in \mathbb{R}$,

$$
\begin{gathered}
d X_{t}=\frac{b-X_{t}}{1-t} d t+d W_{t}, \quad 0 \leq t<1, \\
X_{0}=a .
\end{gathered}
$$

Then the solution of the above SDE is given by

$$
X_{t}=a(1-t)+b t+(1-t) \int_{0}^{t} \frac{d W_{s}}{1-s}, \quad 0 \leq t<1 .
$$

Also,

$$
\lim _{t \rightarrow 1} X_{t}=b, \quad \text { almost surely. }
$$

It is clear that BB satisfies conditions given in (2a)-(2d). It can also be proven that BB satisfies the bounded moment condition, (2e), but we will show that our proposed numerical method can be applied directly to the Brownian bridge process to obtain the improved global error estimate without using the assumption of bounded moments.

In Section 2, we discuss the Euler-Maruyama method and some lemmas used in this paper. In Section 3, we propose the numerical approximation with variable (adaptive) step sizes based on the Euler-Maruyama algorithm and we analyze the error. In Section 4, we apply these methods to approximate the specific example, Brownian bridge, and we show that the order of the global error estimate is better for $\mathrm{BB}$ than for the general case.

\section{Euler-Maruyama Algorithm and Fundamental Lemmas}

Let $W_{t}$ be standard Brownian motion, and let $\mathscr{F}_{t}$ be the natural filtration. We use the following general stochastic differential equation and Itô formula to present the development of Euler-Maruyama algorithm:

$$
d X_{t}=a\left(t, X_{t}\right) d t+b\left(t, X_{t}\right) d W_{t}, \quad X_{0}=\xi
$$

where $a\left(t, X_{t}\right), b\left(t, X_{t}\right) \in[0, \infty] \times \Omega \rightarrow \mathbb{R}$.

The same thing can be given in integral notation assuming all the integrals exist $[9$, p. 22]. The second integral is the Itô integral

$$
X_{t}=X_{0}+\int_{0}^{t} a\left(s, X_{s}\right) d s+\int_{0}^{t} b\left(s, X_{s}\right) d W_{s}
$$

Theorem 1 (Itô Formula $\left[9\right.$, p. 46]). Let $X_{t}$ be an Itô process given by

$$
d X_{t}=a d t+b d W_{t}
$$

where $a:[0, \infty] \times \Omega \rightarrow \mathbb{R}$ and $b:[0, \infty] \times \Omega \rightarrow$ $\mathbb{R}$ are measurable, $\mathscr{F}_{t}$-adapted, and integrable (resp., square integrable) on every interval $[0, t]$, almost surely. Let $g(t, x) \in$ $\mathscr{C}^{2}([0, \infty) \times \mathbb{R})$. Then $Y_{t}=g\left(t, X_{t}\right)$ is again an Itô process and

$$
d Y_{t}=\frac{\partial g}{\partial t}\left(t, X_{t}\right) d t+\frac{\partial g}{\partial x}\left(t, X_{t}\right) d X_{t}+\frac{1}{2} b^{2} \frac{\partial^{2} g}{\partial x^{2}}\left(t, X_{t}\right) d t .
$$

Consider a sequence of times $t_{0}<t_{1}<t_{2}<\cdots<t_{N}$ and the corresponding step sizes $h_{n}=t_{n}-t_{n-1}$. Following [13, p. 
56], we use the Itô formula to expand the drift and diffusion terms in (7),

$$
\begin{aligned}
X\left(t_{n+1}\right) & \\
= & X\left(t_{n}\right)+\int_{t_{n}}^{t_{n+1}} a_{t} d t+\int_{t_{n}}^{t_{n+1}} b_{t} d W_{t} \\
= & X\left(t_{n}\right)+\int_{t_{n}}^{t_{n+1}}\left(a_{t_{n}}+\int_{t_{n}}^{t}(A a)_{s} d s+\int_{t_{n}}^{t}(B a)_{s} d W_{s}\right) d t \\
& +\int_{t_{n}}^{t_{n+1}}\left(b_{t_{n}}+\int_{t_{n}}^{t}(A b)_{s} d s+\int_{t_{n}}^{t}(B b)_{s} d W_{s}\right) d W_{t} \\
= & X\left(t_{n}\right)+a_{t_{n}} h_{n+1}+b_{t_{n}} \Delta W_{n+1}+\int_{t_{n}}^{t_{n+1}} \int_{t_{n}}^{t}(A a)_{s} d s d t \\
& +\int_{t_{n}}^{t_{n+1}} \int_{t_{n}}^{t}(B a)_{s} d W_{s} d t+\int_{t_{n}}^{t_{n+1}} \int_{t_{n}}^{t}(A b)_{s} d s d W_{t} \\
& +\int_{t_{n}}^{t_{n+1}} \int_{t_{n}}^{t}(B b)_{s} d W_{s} d W_{t},
\end{aligned}
$$

where $h_{n+1}=t_{n+1}-t_{n}, \Delta W_{n+1}=W\left(t_{n+1}\right)-W\left(t_{n}\right)$, Af $=$ $f_{1}+a f_{2}+(1 / 2) b^{2} f_{22}, B f=b f_{2}, a_{s}=a(s, X(s))$, and $b_{s}=$ $b(s, X(s))$. Because $W$ is standard Brownian motion, $\Delta W_{n}$ forms a sequence of independent Gaussian random variables with mean zero and $\operatorname{Var}\left(\Delta W_{n}\right)=h_{n}$.

The first three terms of expansion generate the EulerMaruyama algorithm with variable step sizes $h_{n}$ [2, p. 190]. The double integrals yield the local truncation error. So we define the numerical scheme by

$$
Y_{n+1}=Y_{n}+a\left(t_{n}, Y_{n}\right) h_{n+1}+b\left(t_{n}, Y_{n}\right) \Delta W_{n+1} .
$$

With suitable conditions on the coefficient function $a$ and with $b$ constant, it can be shown that mean square local truncation error is of order $O\left(h_{n+1}^{3}\right)$ as $h_{n+1} \downarrow 0$. Let $\epsilon_{n+1}=$ $X_{n+1}-Y_{n+1}$. When step sizes are fixed at $h$, it follows that [13, p. 60]

$$
\mathbb{E}\left[\epsilon_{n+1}^{2}\right] \leq\left(1+m_{1} h\right) \mathbb{E}\left[\epsilon_{n}^{2}\right]+m_{2} h^{3}
$$

It can be further shown that the global error $\left(\mathbb{E}\left[\epsilon_{n}^{2}\right]\right)^{1 / 2}=O(h)$ as $h \downarrow 0$ [13, p. 61]. In the process of estimating the global error, the following lemma is used. This is an easy extension of a fundamental lemma of numerical analysis [8, p. 189].

Lemma 2. If $M_{1} \geq-1$ and $M_{2} \geq 0$ are real numbers and $\left\{a_{n}\right\}_{n=0}^{N}$ is a sequence with $a_{0} \geq 0$ s.t.

$$
a_{n+1} \leq\left(1+M_{1}\right) a_{n}+M_{2}
$$

for all $n=0,1,2, \ldots, N-1$, then

$$
a_{n+1} \leq e^{n M_{1}}\left(\frac{M_{2}}{M_{1}}+a_{0}\right)-\frac{M_{2}}{M_{1}}
$$

for all $n=0,1,2, \ldots, N-1$.

\section{Numerical Approximation for SDE with Singularity}

We return to the SDE of the form (1a)-(1b) presented in Section 1:

$$
\begin{gathered}
d X_{t}=\frac{g\left(t, X_{t}\right)}{1-t} d t+\sigma d W_{t}, \quad 0 \leq t<1, \\
X(0)=x_{0},
\end{gathered}
$$

where $\sigma$ is a constant and $g(t, x)$ satisfies linear growth and Lipschitz conditions with bounds for derivatives up to the second order. In particular, we suppose that, uniformly in $t$ on $[0,1)$,

$$
\begin{array}{r}
\mathbb{E}\left[g\left(t, X_{t}\right)^{2}\right] \leq K_{0}, \\
\mathbb{E}\left[g_{1}\left(t, X_{t}\right)^{2}\right] \leq K_{1}, \\
\left|g_{2}(t, x)\right| \leq C_{1}, \\
\left|g_{22}(t, x)\right| \leq C_{2} .
\end{array}
$$

It follows then that

$$
|g(t, x)-g(t, y)| \leq C_{1}|x-y| .
$$

These conditions follow, for example, from the conditions given in $(2 \mathrm{a})-(2 \mathrm{e})$.

In this case, $a(t, x)=g(t, x) /(1-t)$ and $b(t, x)=\sigma$, and thus

$$
\begin{gathered}
A a=\frac{g(t, x)}{(1-t)^{2}}+\frac{g_{1}(t, x)}{1-t}+\frac{g(t, x) g_{2}(t, x)}{(1-t)^{2}}+\frac{\sigma^{2} g_{22}(t, x)}{2(1-t)}, \\
B a=\frac{\sigma g_{2}(t, x)}{(1-t)}, \\
A b=B b=0 .
\end{gathered}
$$

Note that the derivatives of $a$ are not bounded. Nevertheless, on each subinterval where $t_{n+1}<1$, we have the expansion from (10) and the numerical algorithm from (11), respectively:

$$
\begin{aligned}
X_{n+1}= & X_{n}+\frac{g\left(t_{n}, X_{n}\right)}{1-t_{n}} h_{n+1}+\sigma \Delta W_{n+1} \\
& +\int_{t_{n}}^{t_{n+1}} \int_{t_{n}}^{t}(A a)_{s} d s d t+\int_{t_{n}}^{t_{n+1}} \int_{t_{n}}^{t}(B a)_{s} d W_{s} d t \\
& Y_{n+1}=Y_{n}+\frac{g\left(t_{n}, Y_{n}\right)}{1-t_{n}} h_{n+1}+\sigma \Delta W_{n+1} .
\end{aligned}
$$

Label the two integral terms as $L_{1}$ and $L_{2}$, respectively, and let $\epsilon_{n+1}=X_{n+1}-Y_{n+1}$. We have

$$
\epsilon_{n+1}=\epsilon_{n}+\frac{h_{n+1}}{\left(1-t_{n}\right)} \cdot\left(g\left(t_{n}, X_{n}\right)-g\left(t_{n}, Y_{n}\right)\right)+L_{1}+L_{2} \text {. }
$$


Using the relation

$$
\mathbb{E}\left[\epsilon_{n+1}^{2}\right]=\mathbb{E}\left[\epsilon_{n}^{2}\right]+\mathbb{E}\left[\left(\epsilon_{n+1}-\epsilon_{n}\right)^{2}\right]+2 \mathbb{E}\left[\left(\epsilon_{n+1}-\epsilon_{n}\right) \epsilon_{n}\right]
$$

we have

$$
\begin{aligned}
& \mathbb{E}\left[\epsilon_{n+1}^{2}\right] \\
& \leq \mathbb{E}\left[\epsilon_{n}^{2}\right]+2 \cdot \frac{C_{1}^{2} h_{n+1}^{2}}{\left(1-t_{n}\right)^{2}} \cdot \mathbb{E}\left[\epsilon_{n}^{2}\right]+4 \mathbb{E}\left(L_{1}^{2}\right)+4 \mathbb{E}\left(L_{2}^{2}\right) \\
& \quad+2 \cdot \frac{C_{1} \cdot h_{n+1}}{\left(1-t_{n}\right)} \cdot \mathbb{E}\left[\epsilon_{n}^{2}\right]+2 \mathbb{E}\left[\epsilon_{n} \cdot L_{1}\right]+2 \mathbb{E}\left[\epsilon_{n} \cdot L_{2}\right]
\end{aligned}
$$

We have also used the inequality $\mathbb{E}(X+Y)^{2} \leq 2 \mathbb{E}\left(X^{2}\right)+$ $2 \mathbb{E}\left(Y^{2}\right)$ and the Lipschitz condition (17) here. Since $L_{2}$ is a martingale with respect to the natural filtration, $\mathbb{E}\left[\epsilon_{n} \cdot L_{2}\right]=0$. Now we look at the second moments of the integral terms $L_{1}$ and $L_{2}$. First we use Cauchy-Schwartz inequality twice on $L_{1}$ and the bounds imposed in (16a)-(16d)

$$
\begin{aligned}
& \mathbb{E}\left[L_{1}^{2}\right] \\
& =\mathbb{E}\left[\left(\int_{t_{n}}^{t_{n+1}} \int_{t_{n}}^{t}(A a)_{s} d s d t\right)^{2}\right] \\
& \leq h_{n+1} \cdot \mathbb{E}\left[\int_{t_{n}}^{t_{n+1}}\left(\int_{t_{n}}^{t}(A a)_{s} d s\right)^{2} d t\right] \\
& \leq h_{n+1}^{2} \cdot\left[\int_{t_{n}}^{t_{n+1}} \int_{t_{n}}^{t} \mathbb{E}(A a)_{s}^{2} d s d t\right] \\
& \leq h_{n+1}^{2} \cdot\left[\int _ { t _ { n } } ^ { t _ { n + 1 } } \int _ { t _ { n } } ^ { t } \mathbb { E } \left[\left(\frac{g\left(s, X_{s}\right)}{(1-s)^{2}+\frac{g_{1}\left(s, X_{s}\right)}{(1-s)}}\right.\right.\right. \\
& \leq h_{n+1}^{2} \cdot\left[\int _ { t _ { n } } ^ { t _ { n + 1 } } \int _ { t _ { n } } ^ { t } \left(\frac{g\left(s, X_{s}\right) g_{2}\left(s, X_{s}\right)}{(1-s)^{2}}\right.\right. \\
& \left.\left.\left.+\frac{\sigma^{2} g_{22}\left(s, X_{s}\right)}{2(1-s)}\right)^{2}\right] d s d t\right] \\
& (1-s)^{3} \\
& \left.\left(1-\frac{D_{2}}{(1-s)^{2}}\right) d s d t\right]
\end{aligned}
$$

for some $D_{1}, D_{2}$, and $D_{3}$ that do not depend on $n$ or $h_{n+1}$. It follows that

$$
\begin{aligned}
\mathbb{E}\left[L_{1}^{2}\right] \leq & h_{n+1}^{4} \cdot \frac{D_{1}}{\left(1-t_{n+1}\right)^{4}}+h_{n+1}^{4} \cdot \frac{D_{2}}{\left(1-t_{n+1}\right)^{3}} \\
& +h_{n+1}^{4} \cdot \frac{D_{3}}{\left(1-t_{n+1}\right)^{2}} \leq D_{4} \frac{h_{n+1}^{4}}{\left(1-t_{n+1}\right)^{4}}
\end{aligned}
$$

where $D_{4}$ is independent of $n$ and $h_{n+1}$. Now for $\mathbb{E}\left[L_{2}^{2}\right]$ term we use the Cauchy-Schwartz inequality and the Itô isometry

$$
\begin{aligned}
\mathbb{E}\left[L_{2}^{2}\right] & =\mathbb{E}\left[\left(\int_{t_{n}}^{t_{n+1}} \int_{t_{n}}^{t}(B a)_{s} d W_{s} d t\right)^{2}\right] \\
& =\mathbb{E}\left[\left(\int_{t_{n}}^{t_{n+1}} \int_{t_{n}}^{t} \frac{\sigma \cdot g_{2}\left(s, X_{s}\right)}{(1-s)} d W_{s} d t\right)^{2}\right] \\
& \leq h_{n+1} \cdot\left[\int_{t_{n}}^{t_{n+1}} \mathbb{E}\left(\int_{t_{n}}^{t} \frac{\sigma \cdot g_{2}\left(s, X_{s}\right)}{(1-s)} d W_{s}\right)^{2} d t\right] \\
& \leq h_{n+1}\left[\int_{t_{n}}^{t_{n+1}} \int_{t_{n}}^{t}\left(\frac{\sigma C_{1}}{(1-s)}\right)^{2} d s d t\right] \\
& \leq D_{5} \frac{h_{n+1}^{3}}{\left(1-t_{n+1}\right)^{2}},
\end{aligned}
$$

where $D_{5}$ is independent of $n$ and $h_{n+1}$.

We use Cauchy-Schwartz inequality and the inequality

$$
\mathbb{E}\left[\epsilon_{n}^{2}\right]^{1 / 2} \leq k+k^{-1} \mathbb{E}\left[\epsilon_{n}^{2}\right]
$$

with $k=h_{n+1} /\left(1-t_{n+1}\right)$ to estimate $\mathbb{E}\left[\epsilon_{n} \cdot L_{1}\right]$ :

$$
\begin{aligned}
\mathbb{E}\left[\epsilon_{n}\right. & \left.\cdot L_{1}\right] \\
& \leq \mathbb{E}\left(L_{1}^{2}\right)^{1 / 2} \cdot \mathbb{E}\left(\epsilon_{n}^{2}\right)^{1 / 2} \\
& \leq \sqrt{D_{4}} \frac{h_{n+1}^{2}}{\left(1-t_{n+1}\right)^{2}}\left(\frac{h_{n+1}}{1-t_{n+1}}+\frac{1-t_{n+1}}{h_{n+1}} \mathbb{E}\left[\epsilon_{n}^{2}\right]\right) \\
& \leq D_{6} \frac{h_{n+1}}{1-t_{n+1}} \mathbb{E}\left[\epsilon_{n}^{2}\right]+D_{7} \frac{h_{n+1}^{3}}{\left(1-t_{n+1}\right)^{3}} .
\end{aligned}
$$

For simplicity, we assume $Y_{0}=x_{0}$. For fixed step size, $h_{n}=h=1 / N$, there is no uniform control over the local truncation error since, close to $t=1,1-t_{N-1}=h$. If we fix $\delta>0$ and only consider $t_{n+1}<1-\delta$, then we have the following inequality:

$$
\mathbb{E}\left[\epsilon_{n+1}^{2}\right] \leq\left(1+m_{1} \frac{h}{\delta}\right) \mathbb{E}\left[\epsilon_{n}^{2}\right]+m_{2} \frac{h^{3}}{\delta^{3}},
$$

where $m_{1}$ and $m_{2}$ are some constants which do not depend on $h$ or $\delta$. Using Lemma 2, we have

$$
\mathbb{E}\left[\epsilon_{n}^{2}\right] \leq \exp \left(\frac{m_{1}}{\delta}\right)\left(\frac{m_{2} h^{2}}{m_{1} \delta^{2}}\right) .
$$

For fixed $\delta>0$, this is the original case where the coefficients of the SDE have bounded derivatives. However, if $\delta \downarrow 0$, this estimate shows no decrease in global error unless $h$ decays exponentially relative to $\delta$.

To produce a better estimate and a more efficient algorithm, we consider variable step sizes defined as follows. First fix $h^{*}, 0<h^{*}<1$. Then define step sizes $h_{n}$ and node points $t_{n}$ using $h^{*}$ :

$$
\begin{gathered}
h_{n}=h^{*}\left(1-t_{n-1}\right), \\
t_{n}=t_{n-1}+h^{*}\left(1-t_{n-1}\right) .
\end{gathered}
$$


This implies that

$$
\begin{aligned}
& t_{n}=1-\left(1-h^{*}\right)^{n}, \\
& h_{n}=h^{*}\left(1-h^{*}\right)^{n-1} .
\end{aligned}
$$

This specific definition allows us to estimate the global error using $h^{*}$, and therefore we can control $h^{*}$ to control the global error. Using these step sizes, we have the error estimates

$$
\begin{gathered}
\mathbb{E}\left[L_{1}^{2}\right] \leq D_{4} h^{* 4}, \\
\mathbb{E}\left[L_{2}^{2}\right] \leq D_{5} h^{* 3}, \\
\mathbb{E}\left[\epsilon_{n} \cdot L_{1}\right] \leq D_{6} h^{*} \mathbb{E}\left[\epsilon_{n}^{2}\right]+D_{7} h^{* 3},
\end{gathered}
$$

where $D_{4}, D_{5}, D_{6}$, and $D_{7}$ are positive constants which do not depend on $n$ or $h^{*}$. Using these estimates in (23), we have

$$
\mathbb{E}\left[\epsilon_{n+1}^{2}\right] \leq\left(1+m_{1} h^{*}\right) \mathbb{E}\left[\epsilon_{n}^{2}\right]+m_{2} h^{* 3},
$$

where $m_{1}$ and $m_{2}$ are constants which do not depend on $n$ or $h^{*}$. Using Lemma 2, we have the following.

Theorem 3. Given that the SDE in (15a)-(15b) satisfies the assumptions (16a)-(16d) and $\mathbb{E}\left[\left(x_{0}-Y_{0}\right)^{2}\right] \leq D_{0} h^{* 2}$, then the above constructed algorithm (20) with variable step sizes (32b) has $\mathbb{E}\left[\epsilon_{n}^{2}\right]=O\left(h^{* 2}\right)$, as $h^{*} \downarrow 0$, uniformly in $n$ for $t_{n}<1-\delta<1$, and thus the global pointwise error for the above proposed algorithm is of order $O\left(h^{*}\right)$.

Proof. If we have $N$ steps, (32b) gives $\left(1-h^{*}\right)^{N}=\delta$, and thus

$$
N=\frac{\ln \delta}{\ln \left(1-h^{*}\right)} \leq \frac{-\ln \delta}{h^{*}}, \quad \text { as } h^{*} \downarrow 0 .
$$

Then by using Lemma 2 on (34) and this $N$ we have

$$
\begin{aligned}
\mathbb{E}\left[\epsilon_{n}^{2}\right] & \leq \exp \left(\frac{-m_{1} h^{*} \cdot \ln \delta}{h^{*}}\right) \cdot\left(\frac{m_{2} h^{* 3}}{m_{1} h^{*}}+D_{0} h^{* 2}\right)-\frac{m_{2} h^{* 3}}{m_{1} h^{*}} \\
& \leq \frac{D}{\delta^{m_{1}}} \cdot h^{* 2}
\end{aligned}
$$

where $D$ and $m_{1}$ are constants that do not depend on $n, h^{*}$, or $\delta$.

Corollary 4. Given a positive constant $p<1$ and the SDE (15a)-(15b) satisfying the assumptions in Theorem 3, the above constructed numerical algorithm has global pointwise error $O\left(h^{* p}\right)$, as $h^{*} \downarrow 0$, when $\delta=\left(h^{*}\right)^{2(1-p) / m_{1}}$, where $m_{1}$ is the constant in (34) which only depends on the bounds imposed on $g$ and the moments of the process.

Proof. Letting $\delta=\left(h^{*}\right)^{2(1-p) / m_{1}}$, we have

$$
\mathbb{E}\left[\epsilon_{n}^{2}\right] \leq D h^{* 2 p}
$$

With a simple example, we show that we cannot expect a much better result. Consider the SDE:

$$
d X_{t}=\frac{a}{1-t} d t+\sigma d W_{t}, \quad X_{0}=0,
$$

where $W_{0}=0$. This equation has solution

$$
X_{t}=-a \ln (1-t)+\sigma W_{t}
$$

The variable step size algorithm generates the approximation

$$
Y_{n}=a n h^{*}+\sigma W_{t_{n}}
$$

It follows then that the maximum value of $\left|X_{t_{n}}-Y_{n}\right|=$ an $\left(-\ln \left(1-h^{*}\right)-h^{*}\right)$ occurs at $N$ where $\left(1-h^{*}\right)^{N}=\delta$ and

$$
\left|X_{t_{N}}-Y_{N}\right|=a(\ln \delta)\left(-1-\frac{h^{*}}{\ln \left(1-h^{*}\right)}\right) \sim \frac{a}{2} h^{*} \ln \delta .
$$

Thus if $\delta=h^{* q},\left|X_{t_{N}}-Y_{N}\right| \sim(q a / 2) h^{*} \ln h^{*}$ as $h^{*} \rightarrow 0$ and is thus $o\left(h^{* p}\right)$ for all $p<1$.

\section{Numerical Approximation of Brownian Bridge with Variable Step Sizes}

We apply the above approximation to the Brownian bridge introduced in (3a)-(3b). $g(x)=1-x$ satisfies the Lipschitz and linear growth conditions assumed. We also show that we can relax the bounded moments conditions for this specific case. We use the BB starting at 0 and converging to 1 as $t \rightarrow$ 1. Consider the SDE:

$$
\begin{gathered}
d X_{t}=\frac{1-X_{t}}{1-t} d t+d W_{t}, \quad 0 \leq t<1, \\
X_{0}=0,
\end{gathered}
$$

where $W_{t}$ is the standard Brownian motion. Then using (10) we get

$$
X_{n+1}=X_{n}+\frac{1-X_{n}}{1-t_{n}} h_{n+1}+\Delta W_{n+1}+\int_{t_{n}}^{t_{n+1}} \int_{t_{n}}^{t} \frac{1}{1-s} d W_{s} d t
$$

Note that $(A a)_{s}$ term is zero and that gives us the opportunity to relax the bounded moments condition

$$
\begin{aligned}
A a & =\frac{\partial a}{\partial t}+a \frac{\partial a}{\partial x}+\frac{1}{2} b^{2} \frac{\partial^{2} a}{\partial x^{2}} \\
& =\frac{b-x}{(1-t)^{2}}-\frac{b-x}{(1-t)^{2}}+\frac{1}{2} b^{2} \cdot 0=0 .
\end{aligned}
$$

Using (11) we get

$$
Y_{n+1}=Y_{n}+\frac{1-Y_{n}}{1-t_{n}} h_{n+1}+\Delta W_{n+1}
$$

Now define $\epsilon_{n+1}=X_{n+1}-Y_{n+1}$ to get

$$
\epsilon_{n+1}=\epsilon_{n}-\frac{\epsilon_{n}}{1-t_{n}} h_{n+1}+\int_{t_{n}}^{t_{n+1}} \int_{t_{n}}^{t} \frac{1}{1-s} d W_{s} d t .
$$


Proceeding as before we have that the second moment of the integral term is of order $h^{* 3}$. Now using (22) and analyzing it in the same way as in the previous section, we get

$$
\mathbb{E}\left[\epsilon_{n+1}^{2}\right] \leq \mathbb{E}\left[\epsilon_{n}^{2}\right]+h^{* 2} \mathbb{E}\left[\epsilon_{n}^{2}\right]-2 h^{*} \mathbb{E}\left[\epsilon_{n}^{2}\right]+O\left(h^{* 3}\right) .
$$

For $h^{*}<1$ we get

$$
\mathbb{E}\left[\epsilon_{n+1}^{2}\right] \leq\left(1-h^{*}\right) \mathbb{E}\left[\epsilon_{n}^{2}\right]+O\left(h^{* 3}\right)
$$

which gives the global error $O\left(h^{*}\right)$ using Lemma 2 . Note that, in this case,

$$
\mathbb{E}\left[\epsilon_{n}^{2}\right] \leq D h^{* 2}
$$

for all $n$, independent of $\delta$. However, making $\delta$ small relative to $h^{*}$ increases the number of steps significantly. For example, if we set $\delta=h^{* 2}$, it takes about twice as many steps to reach $1-\delta$ as in the case $\delta=h^{*}$.

For this example, we can verify the error explicitly. Using Algorithm (45), we have

$$
Y_{n}=t_{n}+\sum_{k=1}^{n}\left(1-h^{*}\right)^{n-k} \Delta W_{k} .
$$

Using this with the explicit solution (4), we calculate

$$
\begin{aligned}
\mathbb{E}\left[\epsilon_{n}^{2}\right]= & \left(1-h^{*}\right)^{n}\left(1-\left(1-h^{*}\right)^{n}\right) \\
& \times\left(1+\frac{2 \ln \left(1-h^{*}\right)}{h^{*}}+\frac{1}{1-h^{*}}\right)
\end{aligned}
$$

and thus the maximum value of $\mathbb{E}\left[\epsilon_{n}^{2}\right] \sim h^{* 2} / 12$ as $h^{*} \rightarrow 0$.

\section{Conflict of Interests}

The authors declare that they have no conflict of interests regarding the publication of this paper.

\section{Acknowledgment}

The authors would like to thank the referee for insightful comments and help in improving this paper.

\section{References}

[1] G. Maruyama, "Continuous Markov processes and stochastic equations," Rendiconti del Circolo Matematico di Palermo, vol. 4, pp. 48-90, 1955.

[2] P. E. Kloeden and E. Platen, Numerical Solution of Stochastic Differential Equations, vol. 23 of Applications of Mathematics, Stochastic Modeling, and Applied Probability, Springer, New York, NY, USA, 1992.

[3] P. M. Burrage and K. Burrage, "A variable stepsize implementation for stochastic differential equations," SIAM Journal on Scientific Computing, vol. 24, no. 3, pp. 848-864, 2002.

[4] J. G. Gaines and T. J. Lyons, "Variable step size control in the numerical solution of stochastic differential equations," SIAM Journal on Applied Mathematics, vol. 57, no. 5, pp. 1455-1484, 1997.
[5] N. Hofmann, T. Müller-Gronbach, and K. Ritter, "Optimal approximation of stochastic differential equations by adaptive step-size control," Mathematics of Computation, vol. 69, no. 231, pp. 1017-1034, 2000.

[6] H. Lamba, "An adaptive timestepping algorithm for stochastic differential equations," Journal of Computational and Applied Mathematics, vol. 161, no. 2, pp. 417-430, 2003.

[7] W. Römisch and R. Winkler, "Stepsize control for mean-square numerical methods for stochastic differential equations with small noise," SIAM Journal on Scientific Computing, vol. 28, no. 2, pp. 604-625, 2006.

[8] R. L. Burden, J. D. Faires, and A. C. Reynolds, Numerical Analysis, Weber \& Schmidt, Boston, Mass, USA, 1979.

[9] B. Øksendal, Stochastic Differential Equations: An Introduction with Applications, Springer, Berlin, Germany, 6th edition, 1998.

[10] J. S. Horne, E. O. Garton, S. M. Krone, and J. S. Lewis, "Analyzing animal movements using Brownian bridges," Ecology, vol. 88, no. 9, pp. 2354-2363, 2007.

[11] W. C. Chow, Brownian Bridge, John Wiley \& Sons, WIREs Computational Statistics, New York, NY, USA, 2009.

[12] L. C. G. Rogers and D. Williams, Diffusions, Markov Processes, and Martingales, vol. 2 of Wiley Series in Probability and Mathematical Statistics: Probability and Mathematical Statistics, John Wiley \& Sons, New York, NY, USA, 1987.

[13] J. Gregory and H. R. Hughes, "New general methods for numerical stochastic differential equations," Utilitas Mathematica, vol. 63, pp. 53-64, 2003. 


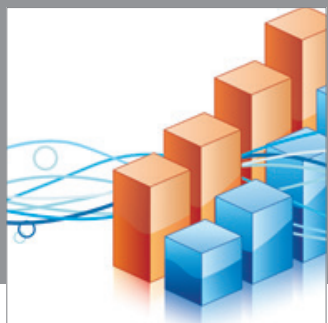

Advances in

Operations Research

mansans

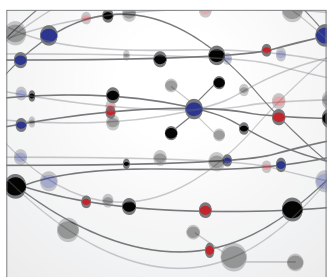

The Scientific World Journal
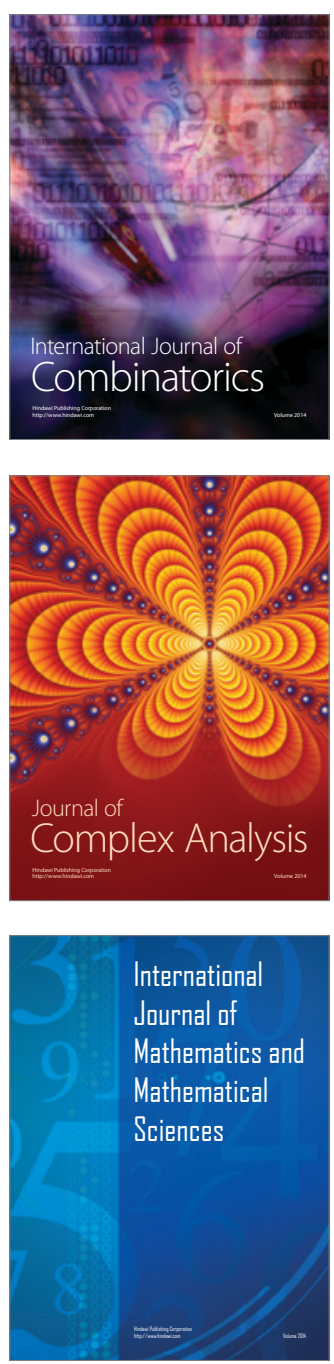
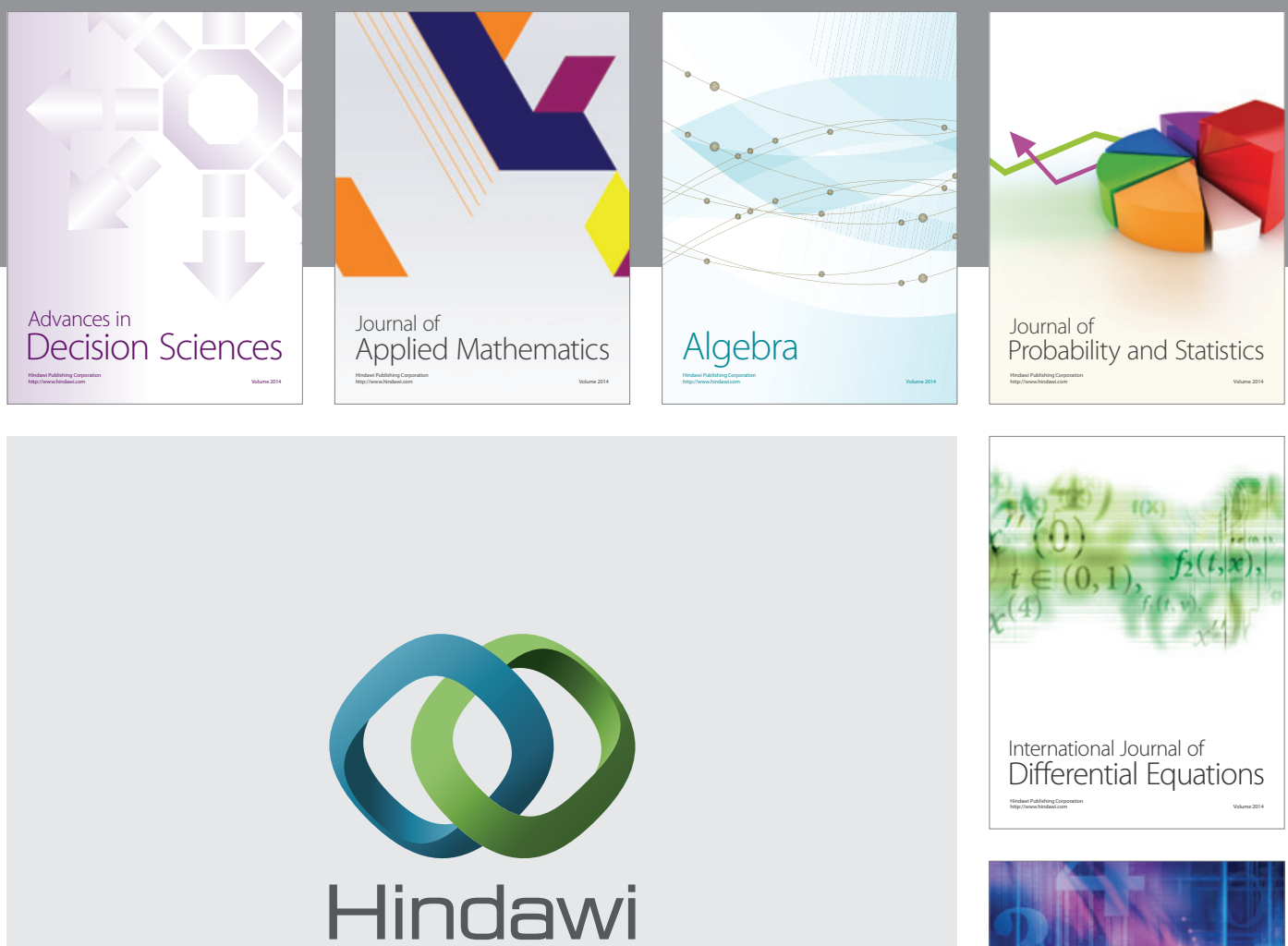

Submit your manuscripts at http://www.hindawi.com
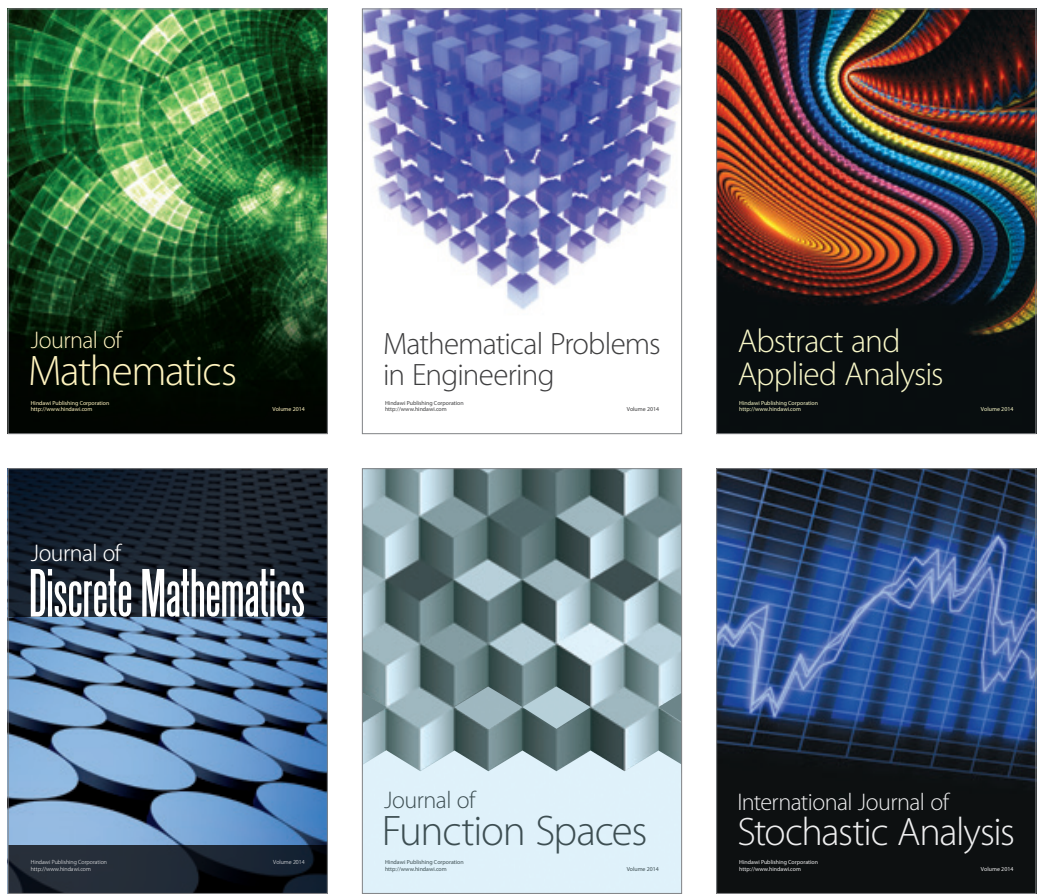

Journal of

Function Spaces

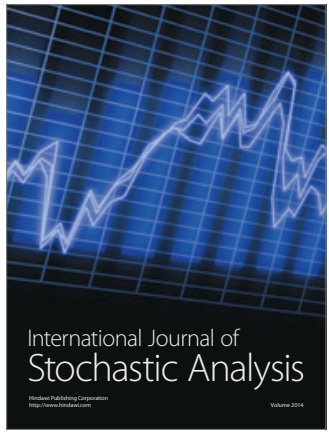

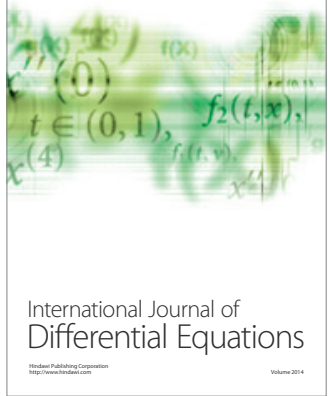
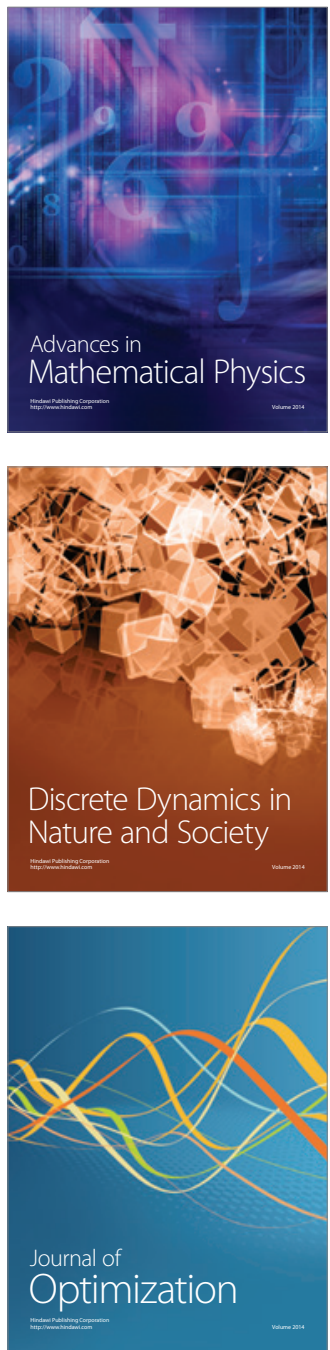